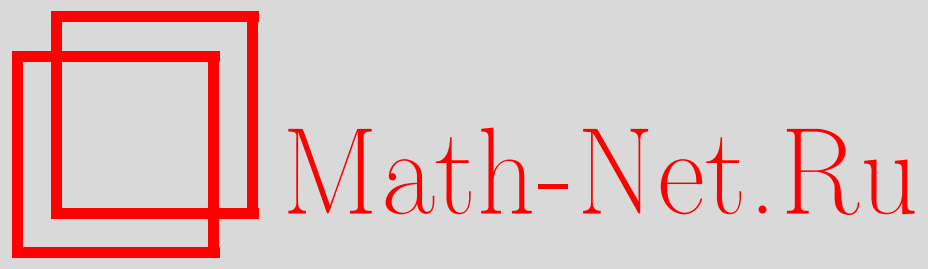

Я. В. Абрамов, Система результантов как набор коэффициентов одного результанта, Функи. анализ и его прил., 2013, том 47, выпуск 3, 82-87

DOI: https://doi.org/10.4213/faa3120

Использование Общероссийского математического портала Math$\mathrm{Net.Ru} \mathrm{подразумевает,} \mathrm{что} \mathrm{вы} \mathrm{прочитали} \mathrm{и} \mathrm{согласны} \mathrm{с} \mathrm{пользователь-}$ ским соглашением

http://www . mathnet.ru/rus/agreement

Параметры загрузки:

IP : 54.237 .59 .107

26 апреля 2023 г., 03:37:12

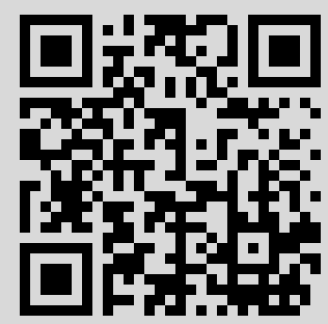


УДК 512.718

\title{
Система результантов как набор коэффициентов одного результанта*
}

\author{
(C) 2013. Я. В. АБрамов
}

1. Введение. Пусть $\mathbb{k}$ - алгебраически замкнутое поле. Рассмотрим следующую классическую задачу.

Задача. Дана система однородных полиномиальных уравнений:

$$
\left\{\begin{array}{l}
f_{0}\left(x_{0}, \ldots, x_{n}\right)=0 \\
\ldots \ldots \ldots \ldots \ldots \\
f_{m}\left(x_{0}, \ldots, x_{n}\right)=0
\end{array}\right.
$$

$$
m \geqslant n, \quad \operatorname{deg} f_{i}=N_{i}, \quad f_{j}(x)=\sum_{\sum_{i} s_{i}=N_{j}} a_{j, s_{0}, \ldots, s_{n}} x_{0}^{s_{0}} \cdots x_{n}^{s_{n}}, \quad a_{j, s_{0}, \ldots, s_{n}} \in \mathbb{k} .
$$

Как определить, есть ли у нее ненулевое решение в $\mathbb{k}^{n+1}$ ?

В [5] доказано, что наборы многочленов $\left(f_{0}, \ldots, f_{m}\right)$ (каждый из которых рассматривается с точностью до умножения на ненулевую константу), для которых система (1) имеет ненулевое решение, составляют проективное алгебраическое подмногообразие в $\mathbb{P}\left(S^{N_{0}}\left(\mathbb{k}^{n+1}\right)\right) \times \cdots \times \mathbb{P}\left(S^{N_{m}}\left(\mathbb{k}^{n+1}\right)\right)$, а именно, существует конечный набор многочленов $\left\{R_{l}(a)\right\}$ с целыми коэффициентами от наборов коэффициентов $a=\left(\ldots, a_{j, s_{0}, s_{1}, \ldots, s_{n}}, \ldots, a_{k, t_{0}, t_{1}, \ldots, t_{n}}, \ldots\right)$ многочленов $f_{j}$, такой, что каждый многочлен $R_{l}(a)$ однороден по коэффициентам каждого многочлена $f_{j}$ и у системы (1) есть ненулевое решение тогда и только тогда, когда $R_{l}(a)=0$ для любого $l$.

Мы будем называть это многообразие результантным многообразием.

Определение 1. Набор многочленов $\left\{R_{l}(a) \in \mathbb{Z}\left[a_{j, s}\right]_{j, s}\right\}$, множество общих нулей которого в $\mathbb{P}\left(S^{N_{0}}\left(\mathbb{k}^{n+1}\right)\right) \times \cdots \times \mathbb{P}\left(S^{N_{m}}\left(\mathbb{k}^{n+1}\right)\right)$ есть результантное подмногообразие, называется однородной системой результантов.

Пример 1.1. Пусть $\operatorname{deg} f_{j}=1, j=0, \ldots, m, f_{j}(x)=\sum_{i} a_{j i} x_{i}$. Тогда однородная система результантов - это множество максимальных миноров матрицы

$$
\left(\begin{array}{ccc}
a_{00} & \ldots & a_{0 n} \\
\vdots & \ddots & \vdots \\
a_{m 0} & \ldots & a_{m n}
\end{array}\right) .
$$

В частном случае задачи (1), когда число уравнений равно числу неизвестных, $m=n$, известно (современное изложение см. в [3]), что результантное

* Работа была частично поддержана лабораторией алгебраической геометрии НИУ-ВШЭ, грант РФ 11.G34.31.0023, и грантами РФФИ 10-01-00836, 12-01-33101 и 12-01-31233. 
многообразие неприводимо и задается как множество нулей одного неприводимого многочлена с целыми коэффициентами. Мы будем обозначать этот многочлен через $R\left(f_{0}, \ldots, f_{n}\right) \in \mathbb{Z}[a]$ и называть однородным результантом $n+1$ многочленов от $n+1$ переменных.

Пример 1.2. Пусть $f(x, y)=\sum_{i} a_{i} x^{i} y^{n-i}, g(x, y)=\sum_{i} b_{i} x^{i} y^{m-i}, a_{0} \neq 0$, $b_{0} \neq 0$. Тогда

$$
R(f(x, y), g(x, y))=\operatorname{Res}(f(1, z), g(1, z)),
$$

где Res - это хорошо известный детерминант Сильвестра

$$
\underbrace{\operatorname{det}\left(\begin{array}{ccccccc}
a_{0} & a_{1} & \ldots & a_{n} & & & \\
& a_{0} & a_{1} & \ldots & a_{n} & & \\
& & \ddots & \ddots & & \ddots & \\
& & & a_{0} & a_{1} & \ldots & a_{n} \\
b_{0} & b_{1} & \ldots & b_{m} & & & \\
& b_{0} & b_{1} & \ldots & b_{m} & & \\
& & \ddots & \ddots & & \ddots & \\
& & & b_{0} & b_{1} & \ldots & b_{m}
\end{array}\right)}_{m+n}\} n
$$

Пусть $X$ - проективное многообразие размерности $n$. Пусть $L_{0}, \ldots, L_{m}$ $(m \geqslant n)$ - линейные расслоения на $X$. Пусть $F_{j} \in H^{0}\left(X, L_{j}\right), j=0, \ldots, m$. Рассмотрим систему

$$
\left\{\begin{array}{l}
F_{0}(x)=0 \\
\cdots \ldots \ldots \\
F_{m}(x)=0
\end{array}\right.
$$

В [3] доказано, что в случае, когда $m=n$ и все $L_{i}$ очень обильны, наборы сечений $\left(F_{0}, \ldots, F_{n}\right)$ (каждое из которых рассматривается с точностью до умножения на ненулевую константу), для которых система (2) имеет решение, составляют неприводимую замкнутую гиперповерхность в $\mathbb{P}\left(H^{0}\left(X, L_{0}\right)\right) \times \cdots \times$ $\mathbb{P}\left(H^{0}\left(X, L_{n}\right)\right)$, т. е. существует неприводимый многочлен $\mathscr{R}\left(F_{0}, \ldots, F_{n}\right)$ (однородный по координатам на каждом $\left.H^{0}\left(X, L_{j}\right)\right)$ на $\bigoplus_{j=0}^{n} H^{0}\left(X, L_{j}\right)$, такой, что у системы (2) есть решение тогда и только тогда, когда $\mathscr{R}\left(F_{0}, \ldots, F_{n}\right)=0$. Такой многочлен называется смешанным резулътантом сечений $F_{0}, \ldots, F_{n}$ расслоений $L_{0}, \ldots, L_{n}$ и обозначается через $R_{L_{0}, \ldots, L_{n}}\left(F_{0}, \ldots, F_{n}\right)$. По аналогии с однородной системой результантов вводится и смешанная система результантов сечений $F_{0}, \ldots, F_{m}$ расслоений $L_{0}, \ldots, L_{m}$, число $m+1$ которых больше $\operatorname{dim} X+1$ (или системы (2)).

Следующие два примера показывают, почему важно изучать смешанные системы результантов.

Пример 1.3. Пусть $X \subset \mathbb{P}^{n}, Y \subset \mathbb{P}^{m}$ - квазипроективные многообразия и $f: X \rightarrow Y$ - регулярный морфизм. Обозначим через $R_{1}\left(y_{0}, \ldots, y_{m}\right), \ldots$, $R_{N}\left(y_{0}, \ldots, y_{m}\right)$ смешанную систему результантов набора сечений $y_{j} f^{*}\left(s_{i}\right)-$ $y_{i} f^{*}\left(s_{j}\right) \in H^{0}\left(X, f^{*} \mathscr{O}_{\bar{Y}}(1)\right), 0 \leqslant i<j \leqslant m$, для $\left(\begin{array}{c}m+1 \\ 2\end{array}\right)$ одинаковых расслоений $f^{*} \mathscr{O}_{\bar{Y}}(1)$ на многообразии $X$, где $s_{0}, \ldots, s_{m}$ - какой-нибудь базис в $H^{0}\left(\bar{Y}, \mathscr{O}_{\bar{Y}}(1)\right)$, а $\left[y_{0}: \cdots: y_{m}\right]$ - точка в $\mathbb{P}^{m}=\mathbb{P}\left(H^{0}\left(\bar{Y}, \mathscr{O}_{\bar{Y}}(1)\right)^{*}\right)$. Тогда замыкание образа $\overline{f(X)} \subset \mathbb{P}^{m}$ является множеством нулей многочленов $R_{k}\left(y_{0}, \ldots, y_{m}\right), k=1, \ldots, N$. 
Основным результатом настоящей работы является формула, выражающая однородную систему результантов общей системы (1) в виде коэффициентов разложения одного однородного результанта подходящей системы, у которой число уравнений равно числу неизвестных и которая явно строится по данной системе (1).

Благодарности. Я очень благодарен А. Л. Городенцеву и Б. Штурмфельсу за полезные обсуждения в связи с этой статьей.

2. Формулировки результатов о результантах. 2.1. Смешанные системы результантов линейных систем сечений расслоений.

Определение 2. Будем говорить, что расслоение $E$ на $X$ имеет достаточно много сечений, если отображение вычисления $\left.H^{0}(X, E) \rightarrow E\right|_{x}$ сюръективно для каждой точки $x \in X$.

Пусть $L_{0}, \ldots, L_{m}$ и $F_{0}, \ldots, F_{m}$ - те же, что и выше. Рассмотрим такие очень обильные линейные расслоения $B_{0}, \ldots, B_{n}$, что при всех $i, j$ линейные расслоения $C_{i j}=L_{j}^{*} \otimes B_{i}$ имеют достаточно много сечений. Такая система всегда существует, если все $L_{i}$ очень обильны, например $C_{i j}=\bigotimes_{k \neq j} L_{k}, B_{i}=\bigotimes_{k} L_{k}$.

Тогда мы имеем следующую теорему:

Теорема 1. Пусть для каждого $i=0, \ldots, n$ задано подпространство

$$
V_{i} \subset \operatorname{Hom}\left(\bigoplus_{j} L_{j}, B_{i}\right)=\bigoplus_{j} H^{0}\left(X, C_{i j}\right),
$$

такое, что каноническое отображение свертки $V_{i} \otimes \mathscr{O}_{X} \rightarrow \bigoplus_{j} C_{i j}$ сюргективно. Тогда набор коэфбициентов $r_{\omega}\left(F_{0}, \ldots, F_{m}\right)$ смешанного результанта сечений расслоений $B_{0}, \ldots, B_{n}$

$$
R=R_{B_{0}, \ldots, B_{n}}\left(\varphi_{0}\left(F_{0}, \ldots, F_{m}\right), \ldots, \varphi_{n}\left(F_{0}, \ldots, F_{m}\right)\right)=\sum_{\omega} r_{\omega}\left(F_{0}, \ldots, F_{m}\right) \varphi^{\omega},
$$

рассматриваемого как многочлен от $\left(\varphi_{0}, \ldots, \varphi_{n}\right) \in V_{0} \oplus \cdots \oplus V_{n}$, является смешанной системой результантов системы (2).

2.2. Однородные системы результантов полиномиальных систем. Применительно к исходной задаче (1) теорема 1 приводит к следующим результатам. Зафиксируем некоторые неотрицательные целые числа $m_{i}$ и $k_{i j}$, такие, что $m_{i}=k_{i j}+N_{j}$, где $i=0, \ldots, n, j=0, \ldots, m$. Рассмотрим однородные многочлены

$$
A_{i j}(x)=\sum_{\sum_{l} s_{l}=k_{i j}} b_{i, j, s_{0}, \ldots, s_{n}} x_{0}^{s_{0}} \cdots x_{n}^{s_{n}}
$$

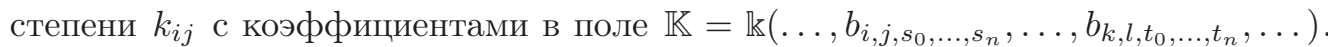
Обозначим набор коэффициентов многочленов $A_{i j}$ через $b=\left(\ldots, b_{i, j, s_{0}, \ldots, s_{n}}, \ldots\right.$, $b_{\left.k, l, t_{0}, \ldots, t_{n}, \ldots\right)}$

Рассмотрим следующий однородный результант $n+1$ многочленов степеней $m_{0}, \ldots, m_{n}$ от $n+1$ неизвестных $x_{0}, \ldots, x_{n}$ с коэффициентами в поле $\mathbb{K}=\mathbb{k}(b)$ :

$$
R\left(\sum_{j=0}^{m} A_{0 j} f_{j}, \sum_{j=0}^{m} A_{1 j} f_{j}, \ldots, \sum_{j=0}^{m} A_{n j} f_{j}\right)=\sum_{\omega} \ell_{\omega}(a) b^{\omega},
$$

где $\ell_{\omega}(a)$ - коэффициенты этого многочлена при одночленах $b^{\omega}$. 
Теорема 2. Коэфбициенты $\ell_{\omega}(a)$ составляют однородную систему результантов системы (1).

Количество результантных уравнений в этой системе равно количеству различных одночленов от переменных $b$ в разложении (3), и этот набор результантных уравнений заведомо избыточен. Его можно уменьшить при помощи следующего обобщения теоремы 2:

Теорема 3. Пусть для каждого $i=0, \ldots, n$ в $\bigoplus_{j=0}^{m} S^{k_{i j}}\left(\mathbb{k}^{n+1}\right)$ задано подпространство $V_{i}$, такое, что для любого $x \in \mathbb{K}^{n+1} \backslash 0$ отображсение вычисления, переводящее набор многочленов $\left(A_{i 0}, A_{i 1}, \ldots, A_{i m}\right)$ в точку $\left(A_{i 0}(x), A_{i 1}(x), \ldots\right.$, $\left.A_{\text {im }}(x)\right) \in \mathbb{k}^{m+1}$, сюргективно отображсает $V_{i}$ на $\mathbb{k}^{m+1}$. Тогда система (1) имеет ненулевое решение в $\mathbb{k}^{n+1}$, если и только если

$$
R=R\left(\sum_{j=0}^{m} A_{0 j} f_{j}, \sum_{j=0}^{m} A_{1 j} f_{j}, \ldots, \sum_{j=0}^{m} A_{n j} f_{j}\right) \equiv 0
$$

как многочлен на $\bigoplus_{i=0}^{n} V_{i}$. Таким образом, коэффициенты многочлена $R$ в каком-либо базисе пространства $\bigoplus_{i=0}^{n} V_{i}$ образуют однородную систему результантов системь (1).

Пример 2.1. Примером набора $V_{i}$, удовлетворяющего условию теоремы 3 , является $V_{i}=\bigoplus_{j}\left\{\sum_{l} a_{l} x_{l}^{k_{i j}} \mid a_{l} \in \mathbb{k}\right\}$.

Следствие. Пусть $\operatorname{deg} f_{0} \geqslant \operatorname{deg} f_{1} \geqslant \cdots \geqslant \operatorname{deg} f_{m} u k_{i j}=\operatorname{deg} f_{i}-\operatorname{deg} f_{j}, u$ пусть для каждого $i=0, \ldots, n$ в $\bigoplus_{j=n+1}^{m} S^{k_{i j}}\left(\mathbb{k}^{n+1}\right)$ задано подпространство $V_{i}$, такое, что для любого $x \in \mathbb{k}^{n+1} \backslash 0$ отображение вычисления, переводящее набор многочленов $\left(A_{i, n+1}, A_{i, n+2}, \ldots, A_{i, m}\right)$ в точку $\left(A_{i, n+1}(x), A_{i, n+2}(x), \ldots\right.$, $\left.A_{i, m}(x)\right) \in \mathbb{k}^{m-n}$, сюргективно отображсает $V_{i}$ на $\mathbb{k}^{m-n}$. Тогда система (1) имеет ненулевое решение, если и только если

$$
R\left(f_{0}+\sum_{j=n+1}^{m} A_{0 j} f_{j}, f_{1}+\sum_{j=n+1}^{m} A_{1 j} f_{j}, \ldots, f_{n}+\sum_{j=n+1}^{m} A_{n j} f_{j}\right) \equiv 0
$$

как многочлен на $\bigoplus_{i=0}^{n} V_{i}$.

Замечание. В работе [2] рассмотрен аналогичный метод построения однородных систем результантов как набора коэффициентов одного результанта для случая двух однородных переменных. Получаемая система результантов состоит из меньшего количества многочленов, чем получилось у нас.

3. Доказательства. Теоремы 2 и 3 следуют из теоремы 1.

Доказательство теоремы 1. Предположим противное. Для $x \in X$ положим

$$
H_{i, x}=\left\{\left(A_{i j}\right)_{j} \in V_{i} \mid \sum_{j=0}^{m} A_{i j}(x) \otimes f_{j}(x)=0\right\}
$$

и $H_{x}=\bigoplus_{i=0}^{n} H_{i, x}$. Условие $R \equiv 0$ равносильно тому, что $\bigoplus_{i=0}^{n} V_{i}=\bigcup_{x \in X} H_{x}$. Если $x$ не является решением системы $(2)$, то $H_{i, x}$ - это гиперплоскость, задаваемая одним ненулевым линейным уравнением (см. (4)) в $V_{i}$ и $H_{x}$ имеет коразмерность $n+1$ как линейное подпространство в $V=\bigoplus_{i=0}^{n} V_{i}$. Если система (2) не имеет решения, то $V$ является объединением $n$-параметрического семейства подпространств коразмерности $n+1$. Противоречие. 
4. Задача о том, когда одна орбита лежит в замыкании другой. Пусть $G$ - неприводимая аффинная алгебраическая группа, $X \subset \mathbb{k}^{l}-$ квазиаффинное алгебраическое многообразие и имеется действие $G: X$.

В работе [4] представлен алгоритм выяснения, когда одна орбита действия группы $G$ лежит в замыкании другой, для случая, когда $X-$ конечномерное линейное представление группы $G$. Здесь мы представим аналогичный алгоритм для общего случая. Этот алгоритм, например, позволяет определить, когда две точки лежат на одной орбите. В самом деле, $y \in G x$ тогда и только тогда, когда $y \in \overline{G x}$ и $x \in \overline{G y}$.

Как следует из существования разложения Леви, $G$ как многообразие изоморфно $G_{\mathrm{red}} \times \mathbb{k}^{r}$, где $G_{\mathrm{red}}-$ редуктивная группа. Из разложения Брюа следует, что $G_{\text {red }}$ содержит в себе открытое плотное подмножество, изоморфное $\left(\mathbb{k}^{*}\right)^{n} \times$ $\mathbb{k}^{p}$. Таким образом, $G$ содержит открытое плотное подмножество $U$, изоморфное $\left(\mathbb{k}^{*}\right)^{n} \times \mathbb{k}^{m}$. Пусть $x$ - точка на $X$. Тогда замыкание $\overline{G x}$ орбиты $G x$ совпадает с замыканием множества $U x=\{g \cdot x \mid g \in U\}$. Если $u \in U$, то будем использовать для вектора $u \cdot x \in X \subset \mathbb{k}^{l}$ обозначение $u \cdot x=F(u)=\left(F_{1}(u), \ldots, F_{l}(u)\right)$. Тогда $F_{i}(u)$ - многочлен Лорана от $u \in U \cong\left(\mathbb{k}^{*}\right)^{n} \times \mathbb{k}^{m}$.

Пусть $A$ - выпуклый многогранник с целыми вершинами в $\mathbb{Z}^{n+m}$ и $M=$ $\left\{m_{1}, \ldots, m_{T}\right\}$ - множество всех целых точек внутри него. Рассмотрим в $\mathbb{P}^{T-1}$ отображение $f_{A}: u \mapsto\left[u^{m_{1}}: \cdots: u^{m_{T}}\right]$. Замыкание его образа $f_{A}(U)$ является торическим многообразием, которое мы обозначим через $X_{A}$. Сечения пучка $\mathscr{O}_{X_{A}}(1)$ можно отождествить с многочленами Лорана с носителями в $A$. Тогда для $\operatorname{dim} A+1$ многочленов Лорана $s_{0}, \ldots, s_{\operatorname{dim}} A$ с носителями в $A$ можно рассмотреть их смешанный результант $R_{A}\left(s_{0}, \ldots, s_{\operatorname{dim} A}\right)$.

Положим $F_{0}(u)=1$. Пусть $A_{i}-$ носитель многочлена $F_{i}(u)$ в $\mathbb{Z}^{n+m}$. Обозначим через $\Delta_{i j}$ для $0 \leqslant i<j \leqslant l$ выпуклую оболочку множества $A_{i} \cup A_{j}$. Пусть $\Sigma_{i j}$ для $0 \leqslant i<j \leqslant l$ - сумма Минковского, $\Sigma_{i j}=\sum_{(s, t) \neq(i, j)} \Delta_{s t}$. Положим $\Delta=\sum_{i<j} \Delta_{i j}$.

Рассмотрим многочлены Лорана $A_{i j, k}(u)=\sum_{t \in \Sigma_{i j}} a_{i j k, t} u^{t}$ для $0 \leqslant i<j \leqslant l$, $k=0, \ldots, \operatorname{dim} \Delta$ с коэффициентами в поле $\mathbb{k}\left(\ldots, a_{i j k, t}, \ldots, a_{p q r, w}, \ldots\right)$.

Положим $y_{0}=1$. Тогда, согласно примеру 1.3 и теореме 1 , замыкание множества $U x$ задается системой уравнений на $y_{1}, \ldots, y_{l}$

$$
R_{\Delta}\left(\sum_{0 \leqslant i<j \leqslant l} A_{i j, 0}(u)\left(y_{i} F_{j}(u)-y_{j} F_{i}(u)\right), \sum_{0 \leqslant i<j \leqslant l} A_{i j, 1}(u)\left(y_{i} F_{j}(u)-y_{j} F_{i}(u)\right), \ldots\right.
$$

$$
\left.\sum_{0 \leqslant i<j \leqslant l} A_{i j, \operatorname{dim} \Delta}(u)\left(y_{i} F_{j}(u)-y_{j} F_{i}(u)\right)\right)=\sum_{\omega} S_{\omega}(y) a^{\omega} \equiv 0 .
$$

5. Пример получаемой однородной системы результантов. Будем считать, что характеристика поля равна 0 или больше 12 . Пусть $n=2$. Для системы вида (1), где все $N_{i}=2$, можно положить $k_{i j}=0$. Тогда однородная система результантов - это набор коэффициентов многочлена

$$
R\left(\sum_{i} a_{i} f_{i}, \sum_{i} b_{i} f_{i}, \sum_{i} c_{i} f_{i}\right)=\sum_{\nu_{a}, \nu_{b}, \nu_{c}} \lambda_{\nu_{a}, \nu_{b}, \nu_{c}}(f) a^{\nu_{a}} b^{\nu_{b}} c^{\nu_{c}} .
$$

Однородный результант трех квадратичных многочленов трех однородных переменных имеет мультистепень $(4,4,4)$, т. е. суммарная его степень равна 12 . 
Обозначим его полную поляризацию через $\widetilde{R}\left(\xi_{1}, \xi_{2}, \xi_{3}, \xi_{4}, \ldots, \xi_{12}\right)$. Тогда однородная система результантов состоит из всевозможных подстановок векторов $\xi_{i}=\left(f_{p_{i}}, f_{q_{i}}, f_{r_{i}}\right)$ в полную поляризацию однородного результанта.

В статье [1] приведено несколько явных выражений для однородного результанта трех многочленов второй степени от трех однородных переменных. Таким образом, в нашем случае можно выписать в явном виде соответствующую систему результантов.

\section{ЛИТЕРАТУРА}

[1] A. Morozov, Sh. Shakirov, New and old results in resultant theory, http://arxiv.org/abs/0911.5278. [2] M. J. Encarnacion, Appl. Algebra Eng. Comm. Comput., 9:3 (1998), 243-245. [3] I. M. Gelfand, M. M. Kapranov, A. V. Zelevinskiy, Discriminants, Resultants, and Multidimensional Determinants, Mathematics: Theory and Applications, Birkhäuser, Boston, 1994. [4] V. L. Popov, Two orbits: When is one in the closure of the other? http://arxiv.org/abs/0808.2735v7. [5] Б. Л. Ван дер Варден, Алгебра, Наука, М., 1979.

Лаборатория алгебраической геометрии НИУ ВШЭ e-mail: zroslav@gmail.com

Поступило в редакцию 30 апреля 2012 г.

УДК 517.956

\section{Об экстремумах зонных функций в периодических волноводах}

\section{(c) 2013. Д. И. Борисов, К. В. ПАНКРАшкин}

Одним их важных вопросов теории периодических дифференциальных операторов является вопрос о наличии лакун в их спектре. Помимо математического интереса, это мотивировано, в частности, приложениями таких операторов к изучению распространения волн в периодических средах. В случае периодических операторов Штурма-Лиувилля классическим является результат о том, что концами спектральных лакун могут служить только периодические и антипериодические собственные значения. В многомерном случае аналогичное свойство часто предполагалось выполненным по умолчанию, более точно, часто подразумевалось, что края лакун должны являться значениями зонных функций при особых значениях квазиимпульса, лежащих на границе либо в центре зоны Бриллюэна. Вместе с тем общая теория не исключает наличия экстремумов зонных функций и при промежуточных значениях квазиимпульса, более того, имеется большое количество физических работ, изучающих так называемый эффект расщепления концов зон (split band edge), т. е. достижение зонными функциями своих экстремальных значений в нескольких внутренних точках зоны Бриллюэна. Это свойство играет важную роль в замедлении волн в фотонных кристаллах, см., например, [2], [4], [5]. Недавно появились работы, обсуждающие расположение экстремумов зонных функций на строгом математическом уровне и содержащие построения соответствующих примеров с привлечением

*Работа первого автора была частично поддержана грантом РФФИ 10-01-00118, Федеральной целевой программой «Научные и научно-педагогические кадры инновационной России на 2009-2013 гг.», контракт 02.740.11.0612, и грантом FCT No. ptdc/mat/101007/2008. 